\title{
Avaliação da alteração do plano oclusal em cirur- gias ortognáticas combinadas com utilização de fixação interna rígida em pacientes com padrão facial de Classe $\|^{*}$
}

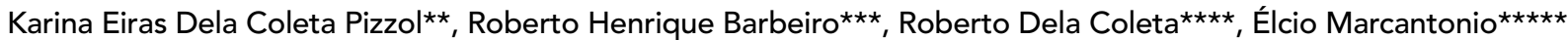

\section{Resumo}

Objetivo: avaliar a estabilidade de cirurgias bimaxilares com fixação interna rígida, na qual promoveu-se uma rotação anti-horária da mandíbula e conseqüente redução do ângulo do plano oclusal. Metodologia: foram estudados 15 pacientes com padrão facial de Classe II e deficiência mandibular. Os movimentos cirúrgicos foram avaliados por meio de medidas lineares e angulares realizadas no programa CefX, obtidas de telerradiografias cefalométricas em norma lateral em três diferentes tempos: (T0) pré-operatório, (T1) pós-operatório imediato e (T2) pós-operatório de no mínimo 6 meses. Resultados e Conclusões: os resultados demonstraram que a cirurgia bimaxilar promove mudanças faciais significativas e, principalmente, que a rotação anti-horária da mandíbula com redução do ângulo do plano oclusal mostrou-se estatisticamente estável, sendo uma opção confiável em cirurgias de pacientes com padrão facial de Classe II com deficiência mandibular.

Palavras-chave: Cefalometria. Fixadores internos. Osteotomia de Le Fort. Cirurgia bucal. Oclusão dentária.

\section{INTRODUÇÃO}

Os conceitos atuais de diagnóstico e plano de tratamento, em Ortodontia e Cirurgia Ortognática, têm focado no equilíbrio e harmonia dos traços faciais. Infelizmente, o tratamento ortodôntico, nem sempre leva à correção ou mesmo à manutenção da estética facial, particularmente nos casos em que a discrepância entre as bases ósseas é de grande proporção, o que torna inviável a obtenção de estética, função e estabilidade adequadas.

* Resumo da Dissertação de Mestrado apresentada ao curso de pós-graduação em Ortodontia do Centro de Pesquisas Odontológicas São Leopoldo Mandic.

** Mestre em Ortodontia pelo Centro de Pós-Graduação São Leopoldo Mandic. Doutoranda em Ciências Odontológicas, Área de Ortodontia, Faculdade de Odontologia - UNESP. Professora do Curso de Especialização em Odontopediatria da APCD-Araraquara.

*** Mestre em Patologia pela Universidade de Campinas -UNICAMP. Doutor em Cirurgia e Traumatologia pela UNESP-Araçatuba. Professor assistente da Disciplina de Cirurgia e Traumatologia da UNESP -Araraquara. Professor do curso de Especialização em Implantodontia da FAEPO- UNESP.

**** Especialista em Ortodontia pela APCD-Araraquara. Professor do Curso de Especialização em Ortodontia da FEB. Presidente do Centro de Pesquisa e Tratamento das Deformidades Buco-faciais - CEDEFACE.

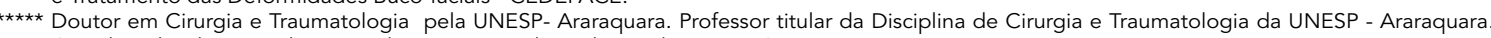
Coordenador do curso de Especialização em Implantodontia da EAP-APCD-Araraquara. 
As deformidades dentoesqueléticas apresentam ampla diversidade de características, no entanto, a Classe II com envolvimento esquelético merece atenção especial, principalmente quando associada ao excesso vertical da maxila e deficiência mandibular. Tal deformidade muitas vezes conhecida como "Síndrome da Face Longa" 34 tem como características: sorriso gengival, terço inferior da face muito longo, com desproporção entre o terço média e inferior da face, mento retrusivo, angulação dos planos oclusal e mandibular aumentada, base nasal estreita, incompetência labial, inclinação do côndilo para trás e proeminência do mento caracteristicamente reduzida ${ }^{2,32}$.

A correção efetiva desta má oclusão é obtida para a maioria dos casos severos através do tratamento ortodôntico-cirúrgico que possibilita o avanço mandibular combinado ao reposicionamento vertical da maxila. Parâmetros para a indicação cirúrgica são: overjet excessivo (maior que $10 \mathrm{~mm}$ ), deficiência mandibular severa (corpo mandibular menor que $70 \mathrm{~mm}$ ), distância perpendicular do pogônio ao násio maior que $18 \mathrm{~mm}$ e altura facial superior a $125 \mathrm{~mm}^{29}$, com exposição excessiva dos incisivos superiores ( $>3 \mathrm{a} 4 \mathrm{~mm}$ ) em repouso e aumento do $1 / 3$ inferior da face ${ }^{15}$.

A evolução de diversas técnicas cirúrgicas na tentativa de reduzir a face longa ${ }^{5}$ permitiu o desenvolvimento da técnica atualmente utilizada de reposicionamento superior da maxila associada ao avanço mandibular, na qual ocorre a autorotação mandibular em direção ântero-superior, corrigindo assim totalmente a má oclusão de Classe II. Segundo Casteigt e Dereudre ${ }^{11}$, a osteotomia Le Fort I realizada em paciente com face longa apresenta tripla vantagem: equilíbrio facial, rotação anti-horária (para cima e para frente) da mandíbula, fechando a mordida aberta, e obtenção da estética facial.

Esta técnica cirúrgica bimaxilar descrita evita a rotação anti-horária isolada da mandíbula, contraindicada por autores como Bell, Proffit, White ${ }^{4}$ e Turvey et al..$^{38}$, uma vez que o efeito biomecânico da rotação mandibular altera o tônus dos músculos mastigatórios e supra-hióideos, resultando em tensão muscular e dos outros tecidos da face, aumentando assim a possibilidade de recidiva. Por sua vez, quando o movimento de intrusão da maxila é realizado em associação ao avanço mandibular há uma redução da tensão destes músculos mastigatórios. Embora alguns autores como Proffit, Bel126; Epker e Fish ${ }^{14}$ considerem a alteração cirúrgica do plano oclusal, que ocorre nesta técnica cirúrgica bimaxilar, como sendo recidivante e que portanto deve ser evitada, autores como Wolford, Chamello e Hilliard ${ }^{44}$ mostraram que a rotação para cima e para frente do plano oclusal é estável, desde que alguns fatores sejam respeitados, como: adequado planejamento e execução do tratamento ortodôntico, correta execução da cirurgia, integridade e estabilidade das ATMs, além de ser um movimento necessário para a obtenção de modificações faciais adequadas ${ }^{12,43,44}$.

De maneira geral, inúmeros autores como Bichara, Chu, Jacobsen ${ }^{6}$; Ellis III, Reynolds e Carlson ${ }^{13}$ consideram que a utilização de fixação interna rígida favorece a estabilidade pós-cirúrgica, em comparação à fixação não rígida, principalmente em casos que requerem movimentos verticais extensos da maxila, grandes avanços mandibulares associados ao movimento rotacional, tornando assim, a recidiva do avanço mandibular menos dependente da quantidade de movimento cirúrgico.

Apesar das inúmeras vantagens, existem alguns fatores que podem contribuir com a recidiva cirúrgica da técnica descrita, tais como: mau posicionamento do segmento proximal, deslocamento do côndilo da fossa articular, movimento de rotação anti-horária do segmento distal, magnitude do avanço mandibular e atividade da musculatura supra-hióidea, erros gnatológicos na obtenção do splint cirúrgico, falha no diagnóstico por análise insuficiente do tecido mole, Ortodontia pré-cirúrgica inadequada, compensações dentárias e falta de proporcionalidade entre a estética do nariz e do lábio ${ }^{8,33,36,41}$. 
Embora o avanço mandibular para correção da Classe II seja objetivo de vários estudos, ainda não há um consenso na literatura sobre a estabilidade de cirurgias bimaxilares com alteração do plano oclusal, o que despertou-nos o interesse em pesquisar este assunto.

\section{PROPOSIÇÃO}

Foi propósito deste estudo:

a) Avaliar a estabilidade de cirurgias combinadas associadas ou não à genioplastia de avanço, em pacientes com padrão facial de Classe II e alteração do plano oclusal.

b) Avaliar a estabilidade da fixação interna rígida em cirurgias bimaxilares.

c) Avaliar se a redução do ângulo do plano oclusal interfere na estabilidade dos resultados.

d) Avaliar a projeção do mento após a redução do ângulo do plano oclusal.

\section{MATERIAL E MÉTODO}

\section{Amostra}

A amostra utilizada no presente estudo foi composta por 15 pacientes, 12 do gênero feminino e 3 do gênero masculino, provenientes do Centro de Pesquisa e Tratamento das Deformidades Buco-Faciais - CEDEFACE. A faixa etária dos pacientes variava entre 15 e 38 anos, com média de idade de 25 anos.

Os critérios para a seleção dos pacientes foram os seguintes:

1) Leucodermas adultos com padrão facial de Classe II, avaliados por meio da análise facial, cirurgia de modelos e radiografias.

2) Tratamento ortodôntico na fase pré e póscirúrgica, submetidos à osteotomia Le Fort I, para reposicionamento superior da maxila e avanço e rotação anti-horária da mandíbula pela técnica da osteotomia sagital bilateral, associada à genioplastia, quando indicada.

3) Redução cirúrgica do ângulo do plano oclusal.

4) Utilização de fixação interna rígida com mini- placas de titânio (Prolig) e os respectivos parafusos na maxila (sistema $2 \mathrm{~mm}$ ) e parafusos na mandíbula (parafusos posicionais, $2 \mathrm{~mm}$ - Sintex).

5) Ausência de outras deformidades craniofaciais, síndromes ou fissuras labiopalatais.

6) Dentados.

7) Bom estado geral de saúde.

Para cada indivíduo, foram feitas telerradiografias cefalométricas em norma lateral no pré-operatório, pós-operatório imediato (aproximadamente 7 dias) e pós-operatório mínimo de 6 meses. Os pacientes foram operados pela mesma equipe cirúrgica. Quando necessário, foram utilizados elásticos ortodônticos para correção de pequenas irregularidades e direcionamento da oclusão, e não como método de fixação. Na maioria dos casos os pacientes receberam alta hospitalar após 48 horas do procedimento cirúrgico e reiniciaram o tratamento ortodôntico após 45 dias.

\section{Telerradiografia e cefalograma}

Para a avaliação, foram utilizadas telerradiografias cefalométricas em norma lateral nos períodos pré-operatório (T0), pós-operatório imediato (aproximadamente 1 semana após a realização da cirurgia - T1) e pós-operatória final (mínimo de 6 meses após o tratamento cirúrgico - T2). As telerradiografias cefalométricas foram obtidas em um mesmo aparelho, conforme o protocolo do Serviço de Radiologia da Disciplina de Radiologia da Faculdade de Odontologia de Araraquara, da Universidade Estadual Paulista "Júlio de Mesquita Filho" - UNESP.

Os traçados das estruturas anatômicas foram construídos de acordo com a metodologia utilizada por Langlade ${ }^{23}$, na qual os desenhos das estruturas da base do crânio e outras áreas da radiografia pré-operatória, foram transferidos para as demais radiografias do mesmo paciente. Assim, pôde-se minimizar erros de reprodutibilidade das estruturas que não sofrem modificação com o ato cirúrgico.

As radiografias foram digitalizadas por meio de 
um scanner da marca Agfa (SNAPSCAN 1236), utilizando-se o software Adobe 5.5 e suas imagens transferidas em 1.200 dpi a um computador para posterior marcação dos pontos cefalométricos. Para a digitalização dos pontos, foi utilizado o software CefX, desenvolvido em Microsoft Visual Basic, utilizando como base de cálculo precisão dupla com 16 algarismos decimais.

Para a obtenção das medidas cefalométricas, as três radiografias de cada paciente foram calibradas por meio do programa CefX, pela distância entre os pontos Sela-Násio da radiografia pré-operatória imediata. $\mathrm{O}$ valor obtido para tal distância foi então reproduzido para as duas radiografias restantes, de forma a minimizar as possibilidades de pequenas distorções, capazes de interferir na obtenção e confiabilidade das medidas cefalométricas. Segundo Jacobson ${ }^{22}$, a digitalização de pontos cefalométricos permite o cálculo preciso de medidas lineares e angulares.

O cefalograma foi composto pelos seguintes pontos de referências, os quais são extensamente utilizados na literatura: ENA; ENP; A; S; N; Pg; PR; B; Ar; ponto posterior de Downs (ponto médio da distância entre as cúspides mesiais dos primeiros molares, superior e inferior); ponto anterior de Ricketts (ponta da cúspide vestibular do primeiro pré-molar inferior ou do primeiro molar inferior decíduo) (Fig. 1).

Outros pontos de referência, requeridos pelo programa, foram utilizados para a digitalização das imagens radiográficas e para a construção do traçado cefalométrico, dando assim, uma visão espacial do desenho das estruturas anatômicas.

No presente trabalho, optou-se por não utilizar o ponto mentoniano (Me), uma vez que o mesmo normalmente está localizado na área em que é realizada a osteotomia do mento. Considerando-se que 10 pacientes da amostra foram submetidos à genioplastia, tal ponto também poderia sofrer remodelação óssea, dificultando a localização do mesmo.

Já em relação ao ponto gônio (Go), este foi substituído pelo ponto PR (Posterior do Ramo ponto mais posterior do bordo posterior do ramo mandibular), pelo fato do ponto Go localizar-se na área da osteotomia sagital da mandíbula (sofrendo rotineiramente remodelação), podendo assim ser confundido com recidiva, além de altamente susceptível a falhas na localização.

Já em relação ao ponto articular (Ar), embora o mesmo seja obtido por meio da intersecção de duas estruturas anatômicas diferentes, sendo portanto mais susceptível a erros de localização, vale ressaltar que no presente estudo, a base do crânio foi transferida da radiografia pré-operatória (T0) para as demais radiografias do mesmo paciente, minimizando assim eventuais erros na marcação deste ponto.

As linhas e planos do traçado cefalométrico utilizado foram baseados nos trabalhos de Burstone et al. ${ }^{9}$; Satrom, Sinclair e Wolford ${ }^{31}$, os quais utilizaram duas linhas de referência, uma vertical e outra horizontal. Entretanto para este trabalho, foi criada uma cefalometria individualizada deno-

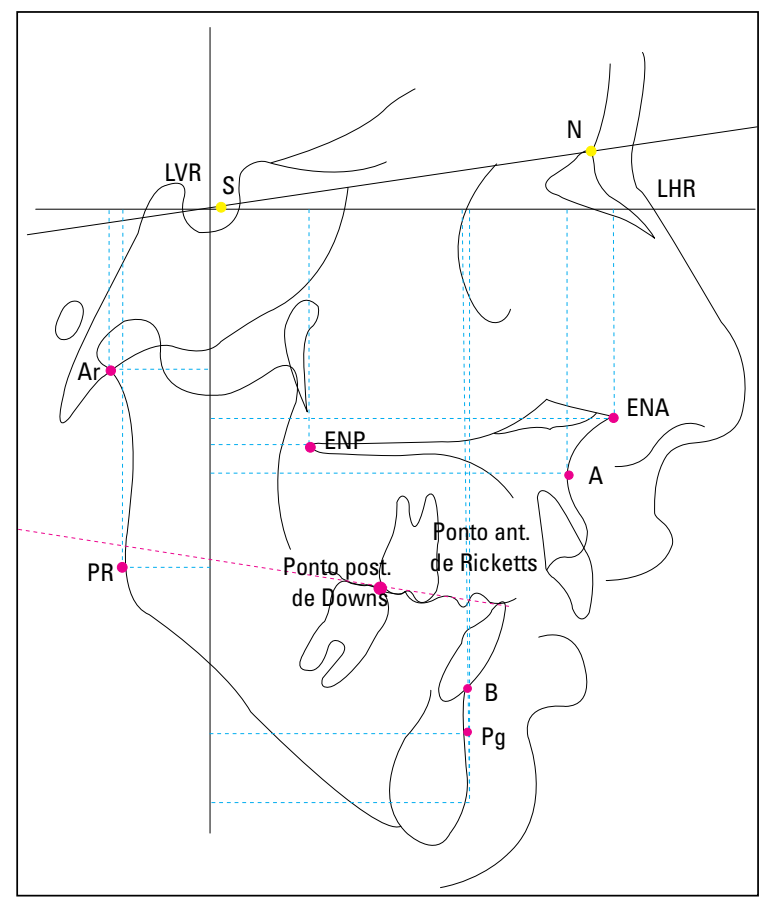

FIGURA 1 - Cefalograma contendo os pontos, as linhas e os planos utilizados na metodologia. 
minada CEDEFACE, que atendesse às necessidades do propósito do estudo.

Linhas de referência utilizadas (Fig. 1):

- SN: linha que passa pelos pontos S e N;

- LHR: linha horizontal de referência (tomada a $-7^{\circ}$ da linha Sela-Násio, partindo do ponto Sela);

- LVR: linha vertical de referência (traçada perpendicularmente à linha horizontal de referência, partindo de Sela).

Determinados os pontos cefalométricos e as linhas e planos de referência, foram realizadas as mensurações lineares em milímetros, e as angulares em graus. A distância em milímetros de cada ponto foi medida perpendicularmente às linhas de referência (eixos $\mathrm{X}$ e $\mathrm{Y}$ ), dessa forma, obteve-se uma medida horizontal e outra vertical de cada ponto em relação aos eixos X e Y (Fig. 1).

Com base nos pontos anatômicos e linhas de referência, foram obtidas as medidas apresentadas na tabela 1 .

As medidas angulares foram obtidas através da mensuração dos ângulos formados pelo Plano Oclusal (PlO) e a linha vertical de referência (LVR) e do Plano Oclusal (P1O) em relação ao Plano Mandibular (PIM).

Uma vez calculadas as distâncias de cada ponto em relação às linhas de referências, o programa CefX calculou a diferença entre as distâncias nos

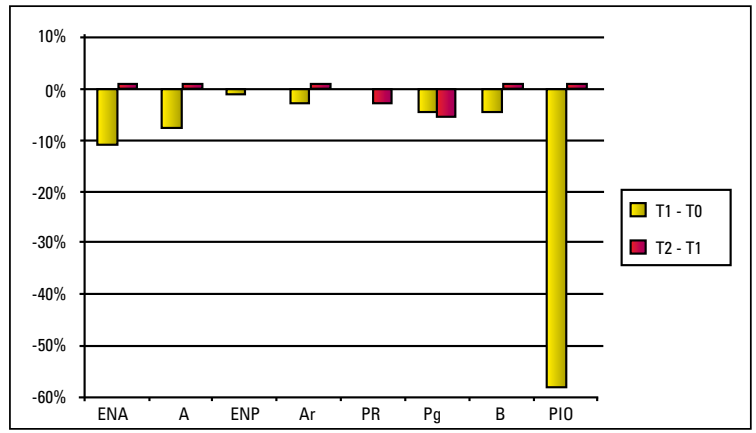

GRÁFICO 1 - Percentual das médias da quantidade do movimento cirúrgico (T1-T0) e da recidiva sobre a medida original (T2-T1), em relação à linha horizontal de referência. Os valores positivos representam 0 aumento percentual da distância de cada ponto até a linha de referência, enquanto os valores negativos representam a redução percentual desta distância.
Tabela 1 - Medidas lineares obtidas a partir da distância de cada ponto cefalométrico em relação às linhas de referência.

\begin{tabular}{cc}
\hline verticais & horizontais \\
\hline ENA-LHR & ENA-LVR \\
ENP-LHR & ENP-LVR \\
A-LHR & A-LVR \\
B-LHR & B-LVR \\
Pg-LHR & Pg-LVR \\
PR-LHR & PR-LVR \\
Ar-LHR & Ar-LVR \\
\hline
\end{tabular}

cefalogramas T0, T1 e T2. Dessa forma, as diferenças entre as medidas, referentes aos mesmos pontos para $\mathrm{Tl}$ e $\mathrm{T} 0$, revelam a quantidade de movimento cirúrgico realizado; e as diferenças das medidas entre $\mathrm{T} 2$ e $\mathrm{T} 1$ quantificam as possíveis mudanças pós-operatórias da estrutura anatômica referentes a cada ponto (Gráf. 1, 2).

Para melhor fidelidade dos resultados, uma vez que a diferença entre as medidas nos períodos $\mathrm{Tl}$ e T2 foi na maioria das vezes menor que $1 \mathrm{~mm}$, em cada radiografia os pontos foram remarcados duas vezes com um intervalo de 1 semana entre cada marcação, de forma que no final fossem obtidos 3 traçados cefalométricos para cada radiografia e calculada uma média aritmética entre os valores.

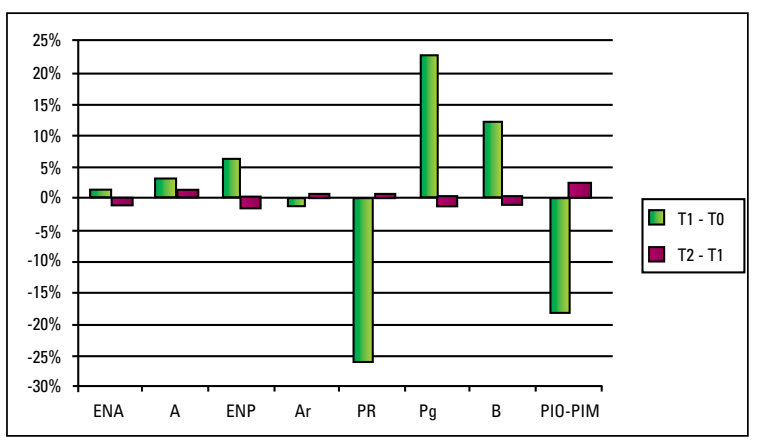

GRÁFICO 2 - Percentual das médias da quantidade do movimento cirúrgico (T1-T0) e da recidiva sobre a medida original (T2-T1), em relação à linha vertical de referência. Os valores positivos representam o aumento percentual da distância de cada ponto até a linha de referência, enquanto os valores negativos representam a redução percentual desta distância. 


\section{Análise estatística}

Os dados foram tabulados utilizando-se o programa "Excel 98" do pacote Office, da Microsoft. Em seguida, os dados foram avaliados estatisticamente por meio da análise de variância ANOVA, nos diferentes tempos: pré-operatório (T0), pósoperatório imediato (Tl) e pós-operatório de no mínimo 6 meses (T2). Nos casos em que o teste " $F$ de Snedecor", que testa a significância entre as variâncias, resultou em probabilidades $(\mathrm{p})$ menores que 0,05 , foi testada a diferença entre as médias por meio do teste de "Tukey-Kramer". Assim, utilizou-se no presente estudo, probabilidades sempre iguais ou inferiores a 0,05 para detectarmos diferenças estatisticamente significantes.

\section{RESULTADO E DISCUSSÃO}

As más oclusões e deformidades dentofaciais, podem ser resultantes de processos patológicos, mas na maioria dos casos são decorrentes de alterações no desenvolvimento normal.

Os pacientes da amostra apresentavam desequilíbrio do sistema estomatognático, neste caso, deformidades dentofaciais com padrão facial de Classe II.

A Classe II esquelética, uma das deformidades dentofaciais com maior incidência na população, é caracterizada por uma alteração distal ou posterior da mandíbula em relação à maxila ${ }^{17}$. Tal deformidade exibe peculiaridades como o corpo mandibular encurtado e o ângulo goníaco relativamente aumentado, no entanto, há a possibilidade da mandíbula ser proporcionalmente adequada, mas estar espacialmente mal posicionada ${ }^{24}$.

Inúmeras variações da Classe II podem estar presentes em um paciente com deformidade esquelética. Destaca-se a "Síndrome da Face Lon$\mathrm{ga}^{\text {"34, }}$ que ganhou status na literatura ortodônticacirúrgica, embora não haja até então um consenso sobre a estabilidade do tratamento de tal deformidade, mesmo que alguns autores ressaltem as inúmeras vantagens em se realizar a osteotomia Le Fort I para intrusão maxilar em pacientes com face longa ${ }^{2,6,11}$.

Entretanto, esta modalidade cirúrgica é relativamente recente no tratamento destes pacientes. Vários estudos de estabilidade cirúrgica na década de 70 concentravam-se no avanço mandibular, usando radiografias cefalométricas em série para identificar os tipos de recidivas e suas causas ${ }^{21,25}$, cabendo ainda salientar que nesse período as osteotomias mandibulares eram praticamente a única modalidade de tratamento das deformidades esqueléticas. Somente após os anos 70 com o aprimoramento das técnicas cirúrgicas, em destaque os trabalhos de Bell, Proffit e White ${ }^{4}$, tornou-se possível a mobilização simultânea da maxila e da mandíbula, permitindo seu reposicionamento nos três planos do espaço. A partir de então, as cirurgias bimaxilares passaram a ser realizadas para corrigir não apenas a deformidade dentária, como também para otimizar a estética da face.

Com o avanço das técnicas cirúrgicas realizadas na maxila ${ }^{5,15}$ e na mandíbula ${ }^{42}$ e da estabilidade proporcionada pela evolução dos métodos de fixação ${ }^{13,33}$, tornou-se possível uma maior estabilidade dos resultados. A utilização da fixação interna rígida em cirurgias combinadas, tem se mostrado um método adequado e previsível e quando comparado à fixação a fios ou fixação intermaxilar, apresenta-se em média mais estável ${ }^{28}$.

Diferentemente da maioria dos estudos embasados em medidas cefalométricas, o diagnóstico dos pacientes da amostra foi baseado na análise facial, na qual as medidas são tomadas diretamente da face do paciente, estando ele em posição natural da cabeça, ou seja, horizontal verdadeira paralela ao solo, dentes em oclusão e lábios em repouso. Embora o Plano de Frankfurt possa ser utilizado como referência em um caso ortodôntico, o recurso da horizontal verdadeira é indispensável quando se trata de deformidades dentofaciais ${ }^{18}$. Portanto, a horizontal verdadeira deve ser utilizada para o diagnóstico, planejamento e controle do tratamento do paciente ortodôntico-cirúrgico, fato este que justificou o uso da linha horizontal 

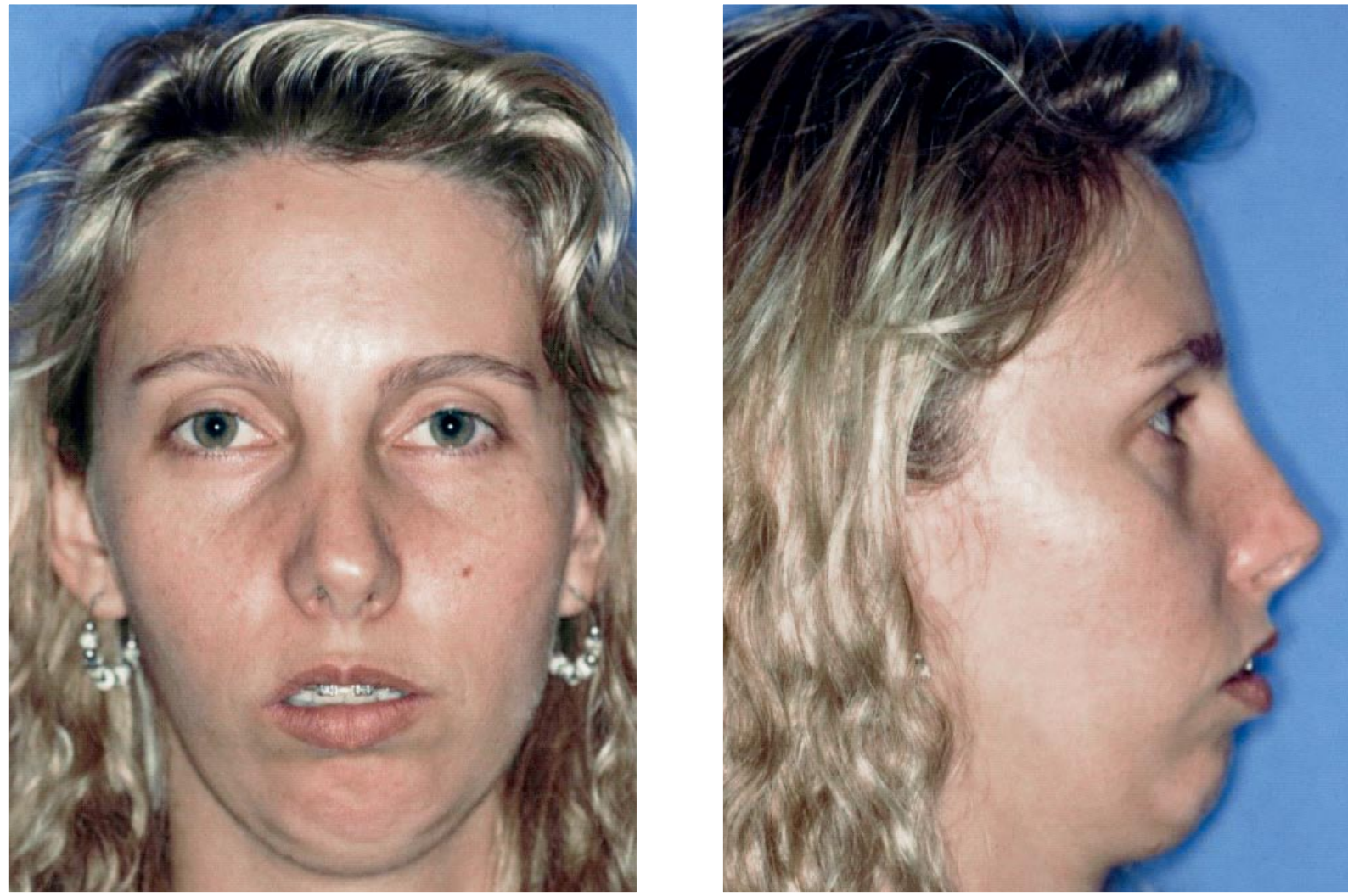

FIGURA 2 - Fotos iniciais da paciente D.I. evidenciando o excesso vertical da maxila, associado à deficiência mandibular ("Síndrome da Face Longa").
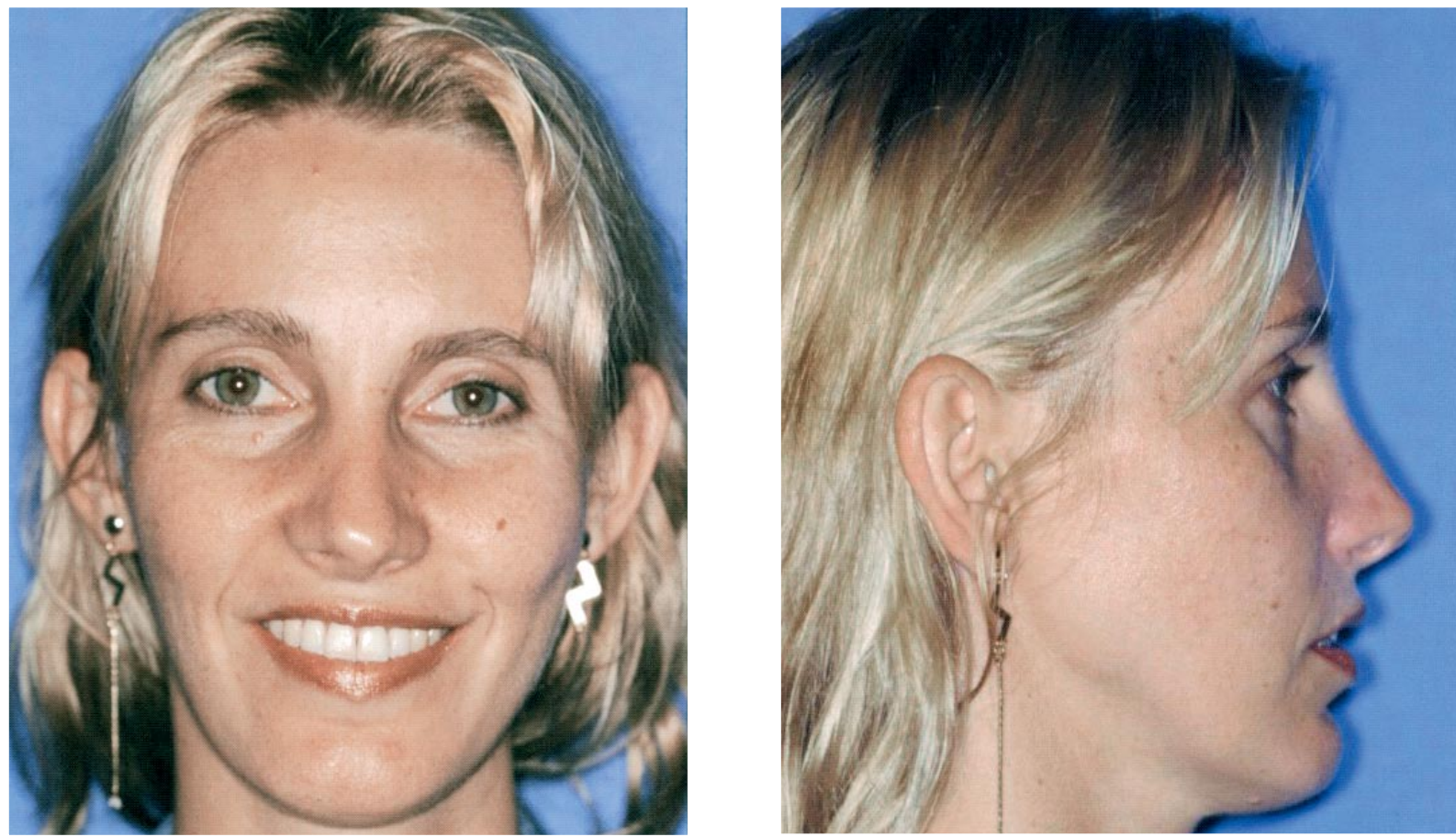

FIGURA 3 - Fotos finais da paciente D.I., evidenciando a melhora significativa da estética facial. 

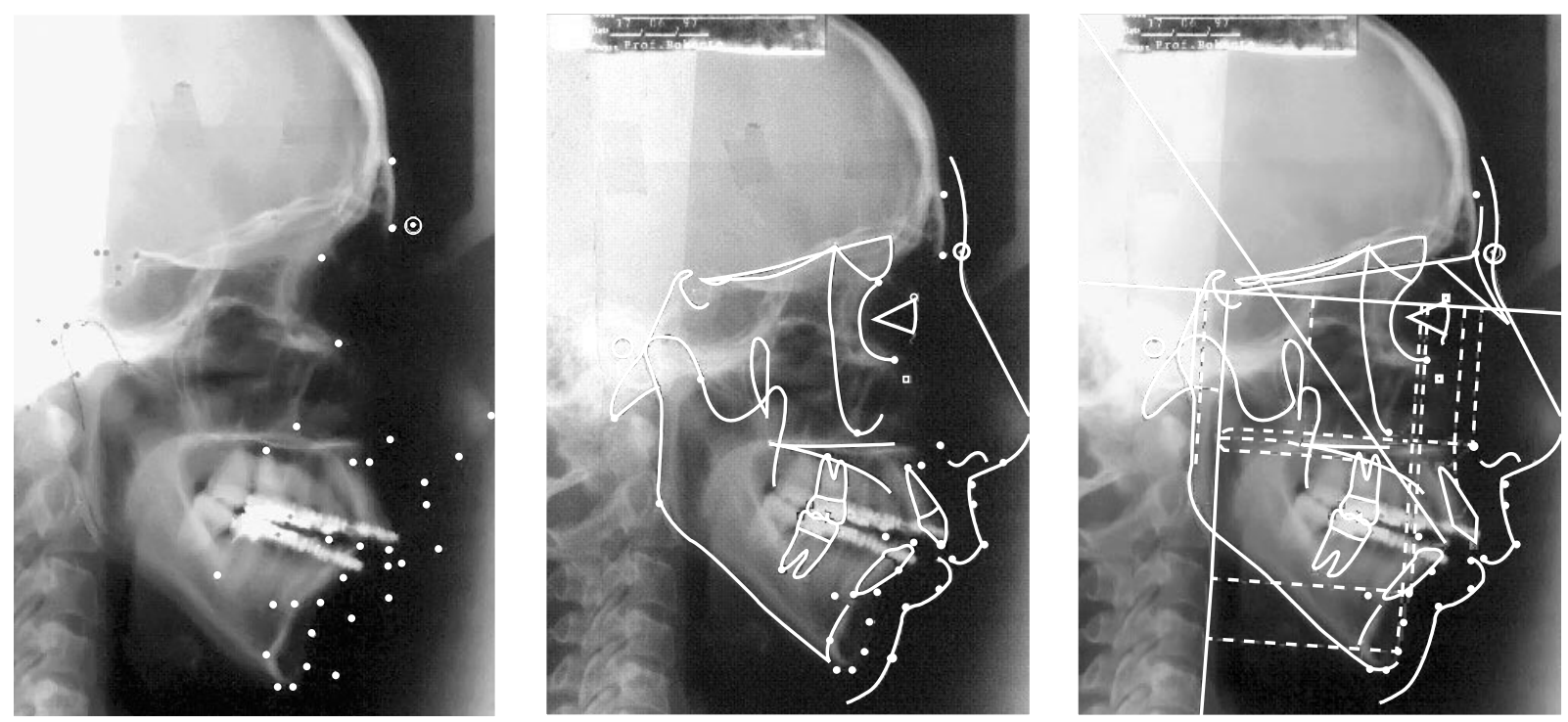

FIGURA 4 - Telerradiografia inicial, mostrando a seqüência desde a marcação dos pontos cefalométricos até a obtenção do traçado.
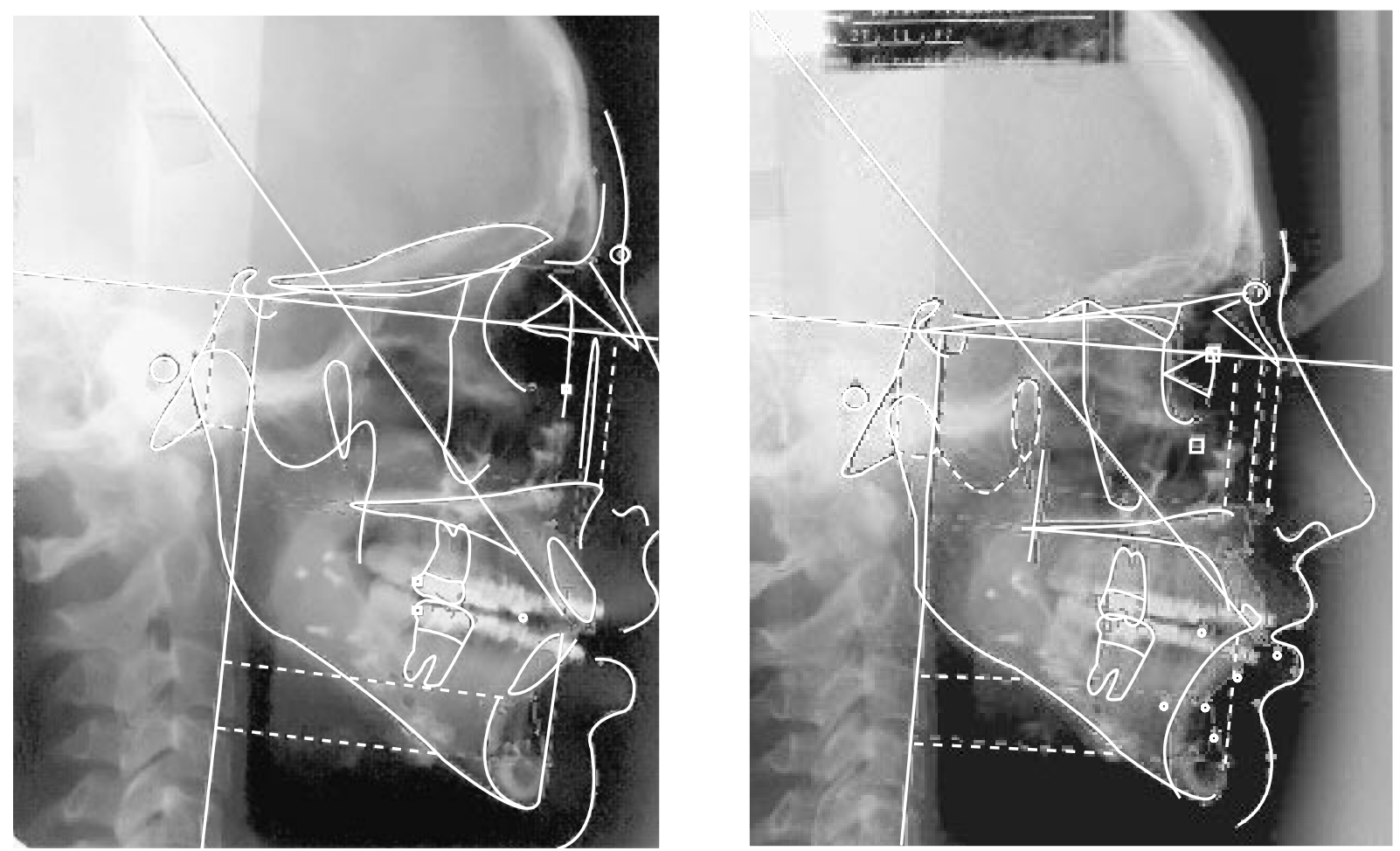

FIGURA 5 - Telerradiografias pós-cirúrgicas (imediata e mínimo de 6 meses), com seus respectivos traçados cefalométricos.

de referência na análise cefalométrica do presente estudo.

Em nossa pesquisa, a análise cefalométrica ser- viu apenas como dado complementar no diagnóstico, entretanto, teve importância primordial na avaliação do movimento cirúrgico realizado, bem 
como na avaliação da estabilidade do mesmo. Para tal, foram utilizadas basicamente medidas lineares (com exceção do ângulo do plano oclusal), que permitem definir as dimensões, as relações posicionais e podem ser transferidas diretamente para os modelos ${ }^{18}$.

Dos 15 pacientes, 12 eram do gênero feminino e apenas 3 do gênero masculino, fato este que pode ser facilmente explicado pela maior procura de pacientes deste gênero pelo tratamento cirúrgico. Dados recentes de uma amostra de 181 pacientes submetidos à cirurgia ortognática pelo CEDEFACE revelam que $64,64 \%$ dos mesmos pertenciam ao gênero feminino. Schendel e Carlotti $\mathrm{Jr}^{32}$ também encontraram maior prevalência deste gênero, representando $88 \%$ da amostra.

Os nossos resultados mostraram que o reposicionamento superior da maxila, em média de $4,25 \mathrm{~mm}$ na região anterior, associado ao giro mandibular, foi responsável por uma redução média de $5 \mathrm{~mm}$ na altura facial anterior, (mínimo de 0,60mm e máximo de $11,19 \mathrm{~mm}$ ). Embora este valor não fosse estatisticamente significante, clinicamente nota-se uma mudança considerável nas características do paciente, muitas vezes suficiente para alterar o padrão facial (Fig. 2-5).

Nossos resultados clínicos comprovam a opinião de Wolford, Chamello e Hilliard ${ }^{43}$, que relatam ser a intrusão maxilar associada à rotação mandibular a única modalidade cirúrgica capaz de alterar dramaticamente o contorno dos tecidos moles e o padrão facial desses pacientes. A combinação desses procedimentos resulta num melhor resultado estético, promovendo uma oclusão mais estável $^{38}$. Além disso, alguns autores sugerem que a utilização da osteotomia maxilar concomitante parece aumentar a estabilidade dos procedimentos mandibulares ${ }^{8}$.

Em concordância com os achados da literatura, a estabilidade do movimento cirúrgico realizado na maxila (ENA e ENP), tanto no plano vertical quanto no horizontal, foi estatisticamente adequada, apresentando recidiva inferior a $0,4 \mathrm{~mm}$ do movimento realizado, tanto para a região anterior quanto para a posterior, provavelmente devido à atenção aos detalhes cirúrgicos e ao uso de fixação interna rígida, uma vez que a mesma, tende a melhorar a estabilidade, eliminando recidivas superiores a $2 \mathrm{~mm}^{31}$. Cabe ainda salientar que alterações superiores a $2 \mathrm{~mm}$ não são causadas por falhas na localização dos pontos cefalométricos e sim pela recidiva propriamente dita ${ }^{40}$.

Os valores da amostra foram semelhantes aos encontrados por Hennes et al. ${ }^{19}$, que observaram apenas $0,1 \mathrm{~mm}$ de recidiva da maxila no plano vertical e $0,7 \mathrm{~mm}$ no plano ântero-posterior e com os achados de Satrom, Sinclair e Wolford ${ }^{31}$ de $0,5 \mathrm{~mm}$ (6\%) e de Van Sickels, Larsen e Trash ${ }^{39}$ com 5\% de recidiva, mostrados para fixação rígida.

Autores como Bishara, Chu, Jacobsen ${ }^{6}$; Stoker, Epker $^{37}$; Proffit, Phillips e Turvey ${ }^{27}$ estudaram diferentes amostras onde observaram pouca ou nenhuma mudança pós-cirúrgico e que esta mudança quando presente foi em direção ao movimento cirúrgico, ocorrendo assim uma intrusão adicional da maxila, sendo na maioria compensatória, resultando em alteração real de menos de $2 \mathrm{~mm}$.

Esta alteração posicional da maxila em direção ao movimento cirúrgico, deve-se em parte pela própria ação muscular mastigatória, que tende a levar a maxila superiormente, ou por contato ósseo pobre, o que causaria uma intrusão adicional da maxila ${ }^{37}$. Este movimento superior da maxila, em média $20 \%$ do movimento total realizado, ocorre no período de fixação intermaxilar, recidivando por completo após a remoção do bloqueio maxilo-mandibular, fornecendo como resultado, um índice muito baixo de recidiva ${ }^{3}$. Vale ainda salientar que este tipo de bloqueio não foi utilizado em nosso estudo. Portanto, a utilização da osteotomia maxilar concomitante tende a aumentar a estabilidade dos procedimentos mandibulares associada à fixação rígida.

Ainda com relação à maxila, é importante destacar que em nosso estudo, o valor relevante observado no movimento ântero-posterior cirúr- 
gico do ponto A (média de 2,30mm), e que não foi estatisticamente significante para a ENA, é justificado pelo fato do reposicionamento superior da maxila ter sido em média mais pronunciado na área da ENA (média de 4,25mm) do que na ENP $(0,5 \mathrm{~mm})$, sendo responsável por uma rotação anti-horária da maxila, projetando o ponto A para anterior e para cima (média de $4,06 \mathrm{~mm}$ ). As medidas lineares da posição de A e B são de grande utilidade para a previsão ortodôntica e cirúrgica, possibilitando a visualização do efeito produzido pela recolocação ântero-posterior de qualquer um desses pontos ${ }^{18}$. Quando se planeja uma mudança na angulação do plano oclusal, é importante que se utilize preferencialmente a ENP como linha de fulcro para a intrusão anterior da maxila, e a ENA nos casos em que se deseja extrusão do segmento posterior. Entretanto, deve-se evitar utilizar o centro da maxila como linha de fulcro, uma vez que esta é de difícil reprodução cirúrgica, podendo levar à instabilidade deste movimento.

No plano vertical, embora a redução da altura facial não tenha sido significante para o ponto $\mathrm{Pg}$, no ponto $B$ observou-se alteração estatisticamente significante (B-LHR), em média de 4,01 mm. Esta diferença encontrada para os pontos $\mathrm{B}$ e $\mathrm{Pg}$ podem ser justificadas pela menor distância entre o ponto $\mathrm{B}$ e a LHR, tornado as pequenas alterações posicionais mais significantes e, principalmente, pelo fato do ponto B ser um ponto dentoalveolar, não sofrendo portanto modificações com a genioplastia. Já em relação ao movimento ântero-posterior da mandíbula, este promoveu uma mudança do comprimento real mandibular que representa um movimento médio de 7,83mm quando medido no ponto B. Quando este movimento é medido no pogônio, temos um avanço médio de $14,07 \mathrm{~mm}$. Embora o valor seja superior a $10 \mathrm{~mm}$, sendo considerado instável ${ }^{30}$, este representa não apenas o movimento puro da mandíbula, mas também está associado à genioplastia de avanço, realizada em 10 pacientes da amostra. Em ambos os pontos de referência, a estabilidade encontrada estava dentro dos parâmetros estatísticos, sendo inferior a $1 \mathrm{~mm}$, valores estes que são comparáveis aos menores encontrados na literatura. Cabe ainda salientar que em todos os casos em que a genioplastia foi realizada, esta deslocou o mento em direção ânterosuperior, potencializando o avanço total da mandíbula, bem como reduzindo a altura facial inferior, devido ao desenho da osteotomia do mento que se apresentava inclinado.

A recidiva de avanços mandibulares descrita na literatura é variáve ${ }^{10} \mathrm{e}$ os fatores citados como responsáveis pela recidiva em cirurgias bimaxilares relacionam-se à tensão da musculatura mastigatória e supra-hióidea, ao deslocamento condilar, rotações mandibulares, mudanças posicionais ou deslocamento entre os segmentos proximal e distal, magnitude do avanço mandibular, ângulo do plano mandibular elevado e falha no diagnóstico $^{1,7,21,25,36}$.

Para Epker, Stella e Fish ${ }^{16}$, há ainda um fator adicional que afeta a estabilidade do reposicionamento superior da maxila e simultâneo avanço mandibular - a quantidade e qualidade do osso na interface posterior da maxila. Duas conseqüências podem ocorrer neste caso: na primeira, a maxila é expandida e, existe contato pobre de osso posteriormente e na segunda, o osso da região posterior é fino e estruturalmente não produz uma interface estável. Quando qualquer um dos fatores está presente, há uma chance potencial da maxila inclinar-se para cima na região posterior e para baixo na região anterior, tendo com conseqüência a rotação horária do mento (recidiva) e/ou rotação do segmento proximal e/ou sobrecarga excessiva condilar.

A falha dos músculos em adaptarem-se completamente às alterações no comprimento, tem sido citada como um importante fator na estabilidade pós-operatória ${ }^{45}$. Em procedimentos cirúrgicos onde o estresse sobre a musculatura mastigatória é elevado, principalmente em grandes avanços, é benéfico, se possível, realizar a separação cirúr- 
gica dos músculos de suas inserções. Além disso, o movimento superior simultâneo da maxila pode minimizar a tensão muscular e, assim, colaborar com a estabilidade nestes casos.

Em alguns casos, o ventre anterior do digástrico e músculo gênio-hióideo podem ser fortes o suficiente para retrair a mandíbula avançada, portanto, os músculos tornam-se contribuintes na recidiva pós-operatória. Sendo assim, a miotomia supra-hióidea deve ser indicada quando a soma total do avanço mandibular e genioplastia, produzem um movimento superior a 30\% do seu comprimento original ${ }^{16}$.

No nosso estudo, 2 pacientes foram submetidos à miotomia supra-hióidea, uma vez que o movimento mandibular total realizado nestes pacientes foi em média de $25,08 \mathrm{~mm}$, representando, em média, $49,85 \%$ do comprimento mandibular original. Portanto, a quantidade de movimento realizado nestes pacientes, justifica a miotomia, uma vez que, nesses casos, nota-se durante a mobilização da mandíbula dificuldade em avançá-la e, conseqüentemente, tensão sobre os músculos supra-hióideos.

De acordo com estudos sobre o reposicionamento condilar após a osteotomia dos maxilares ${ }^{20}$, pode-se afirmar que o deslocamento intra-operatório dos côndilos mandibulares das suas posições funcionais na cavidade glenóide, resulta em uma recidiva esquelética imediatamente à liberação do bloqueio intermaxilar.

Os resultados obtidos em nosso estudo, mostraram que a região condilar representada pelo ponto Ar, sofreu movimentação estatisticamente significante no plano vertical, embora sendo, em média, de $0,91 \mathrm{~mm}$. Mesmo que os côndilos aparentem ter sofrido alteração espacial, estes se apresentaram estáveis dentro da cavidade glenóide (Gráf. 1). No plano horizontal, não foram observadas modificações significantes deste ponto. Quatro hipóteses foram levantadas, que poderiam justificar o deslocamento condilar no plano vertical: 1) seria um erro na localização do ponto cefalométrico, ou seja, na fase pré-operatória e na pós-operatória, o mesmo ponto teria duas marcações espaciais diferentes; 2) uma falha no momento da fixação da mandíbula, deslocando o côndilo da sua posição original na cavidade glenóide; 3) distorções radiográficas por movimentação da cabeça do paciente no eixo sagital, provavelmente causada por falhas na colocação do cefalostato e 4) falha na calibragem do aparelho de raios-X.

Com a finalidade de observar qual dos fatores seria o responsável pela movimentação cirúrgica do côndilo, todas as radiografias foram reavaliadas, bem como suas marcações cefalométricas. Dessa forma, pôde-se observar que os pontos foram corretamente localizados minimizando a possibilidade de erro do examinador. Através da sobreposição dos traçados cefalométricos, preconizada por Jacobson ${ }^{22}$ e Langlade ${ }^{23}$, tornou-se possível verificar a possibilidade do deslocamento condilar. Nenhum caso da amostra apresentou deslocamento do mesmo, entre as fases pré-cirúrgica e pós-cirúrgica, que pudesse ser perceptível visualmente. Sendo assim, a possibilidade de falha na execução da técnica cirúrgica foi descartada. Por fim, observou-se a possibilidade de distorções nas tomadas radiográficas longitudinais. Mesmo com todas as tomadas radiográficas de cada paciente tendo sido submetidas à calibragem pelo programa CefX, temos que as distorções presentes em diferentes áreas da mesma radiografia não são normalmente iguais, uma vez que os raios que produzem a imagem não são paralelos. Portanto, as pequenas alterações presentes na localização do ponto articular no plano vertical foram possivelmente provocadas por distorções das imagens radiográficas, sejam estas causadas pelo próprio aparelho de raios-X ou por movimentação da cabeça do paciente.

Já o ramo mandibular, representado pelo ponto PR, mostrou-se estável no plano vertical, embora tenha apresentado movimentação em direção anterior de $2,21 \mathrm{~mm}$, em média, decorrente da inevitável alteração posicional do ângulo goníaco durante a rotação mandibular, fato este confirmado pela sobreposição dos traçados. Além disso, o 
fato do ponto PR estar localizado próximo à linha vertical da referência, faz com que pequenas alterações espaciais tornem-se estatisticamente significantes. Cumpre salientar que embora a porção posterior do ramo tenha sofrido pequenas modificações no ato cirúrgico, estas, não comprometeram a estabilidade do movimento rotacional da mandíbula, grande responsável pelas modificações clínicas, principalmente do terço inferior da face. A comprovação disto é observada através da estabilidade pós-cirúrgica de todos os pontos cefalométricos (Gráf. 1, 2) e da alteração da disposição dos planos horizontais da face.

O grau de divergência dos planos faciais normalmente fornece informações quanto à direção de crescimento e quanto ao grau de dificuldade encontrada no tratamento da dimensão vertical. Problemas de hiper-divergência esquelética são algumas vezes referidos como "Síndrome da Face Longa"22. Outro método de averiguação é o ângulo do plano ocluso-mandibular (P1O-PlM), no qual é possível a observação da divergência esquelética e, conseqüentemente, do padrão facial ${ }^{35}$. O valor médio para este ângulo, segundo Schudy ${ }^{35}$, é de $7^{\circ}$ a $21^{\circ}$, sendo os valores superiores à média indicadores de hiper-divergência dos planos, assim como o que foi encontrado para os pacientes da amostra, que na fase pré-cirúrgica apresentavam uma média de $35^{\circ}$ para este ângulo, sendo modificada para $28,97^{\circ}$ após o ato cirúrgico, com uma redução mínima de $2,73^{\circ}$ e máxima de $11,72^{\circ}$ (Gráf. 2).

Indiscutivelmente, a grande modificação causada pela cirurgia ocorreu no plano oclusal, em média de $6,10^{\circ}$, o que representa uma alteração de 58\% (Gráf. 1). Segundo Fonseca ${ }^{18}$, a inclinação normal do plano oclusal em relação à horizontal verdadeira é de $8^{\circ}$ com desvio padrão de $\pm 5^{\circ}$. Assim, o plano oclusal que inicialmente era, em média, de $10,46^{\circ}$ em nosso estudo, passou a ser de $4,36^{\circ}$, em média, no pós-operatório, sendo concordante ao relatado pelo autor. As alterações na inclinação do plano oclusal são refletidas especialmente sobre as posições ântero-posterior e vertical do mento.

Para Fonseca ${ }^{18}$, existem duas formas de se obter um novo plano oclusal: 1) por auto-rotação da mandíbula e 2) por alteração seletiva através da criação de um plano oclusal opcional, em osteotomia com separação dos côndilos. Se o plano oclusal diminui, aumentará a projeção ânteroposterior do mento. A alteração da inclinação do plano oclusal não está isenta de riscos, sobretudo quando se diminui sua angulação, a mandíbula gira em sentido anti-horário e podem aparecer sinais de instabilidade articular devido ao aumento de cargas sobre a ATM e o aumento da dimensão do ramo ascendente da mandíbula.

Embora alguns autores considerem a alteração cirúrgica do plano oclusal, mais especificamente o movimento anti-horário do mesmo, como uma modalidade de tratamento recidivante e que deve ser evitada ${ }^{14,26}$, a rotação para cima e para frente do plano oclusal é estável quando corretamente executada e na presença de integridade e estabilidade das ATMs ${ }^{12,44}$.

Portanto, há a necessidade de se avaliar o estado das ATMs antes da cirurgia, particularmente quando se reduz o ângulo do plano oclusal. O movimento associado à redução deste plano, irá aumentar as pressões nas articulações, enquanto a musculatura, os tecidos moles e estruturas dentárias e esqueléticas têm a chance de se readaptarem. Nas articulações íntegras e estáveis, eles podem ser capazes de suportar o aumento de carga durante a fase de adaptação ${ }^{44}$. Assim, é evidente que o aumento ou redução do plano oclusal, quando indicados em cirurgia bimaxilar, podem prover resultados estéticos e funcionais ótimos. Disso podemos afirmar, pelos nossos resultados, que mesmo com uma redução média de 58\% do ângulo do plano oclusal em nossos pacientes, este se mostrou estatisticamente estável e os ganhos estéticos e mudanças faciais foram mantidos (Fig. 2-5). Este estudo, portanto, mostra a estabilidade da redução do plano oclusal com cirurgia bimaxilar com fixação rígida na presença de integridade e estabilidade articular. 


\section{CONCLUSÕES}

Os resultados obtidos, nos limites da metodologia empregada, permitem concluir que:

a) As cirurgias combinadas, de reposicionamento superior da maxila associado ao avanço e rotação anti-horária da mandíbula por meio de osteotomia sagital bilateral e genioplastia de avanço, mostraram ser uma modalidade cirúrgica estatisticamente estável.

b) A fixação rígida tanto da maxila quanto da mandíbula promoveu a estabilidade do movimento cirúrgico. c) A redução significativa do ângulo do plano oclusal não interferiu na estabilidade dos resultados obtidos.

d) A redução do ângulo do plano oclusal, permitiu a obtenção de adequada projeção da mandíbula com um importante ganho estético.

\title{
Evaluation of occlusal plane alteration in Orthognathic Surgeries combined with rigid internal fixation in Class II skeletal facial standard patients
}

\begin{abstract}
Aim: to access the surgical stability combined with rigid internal fixation, where the counter-clockwise jaw rotation was carried out, and consequently the occlusal plane reduction. Methods: fifteen Class II skeletal facial standard patients were studied. The surgical movements were assessed by linear measures and angles accomplished at the CefX program, collected from cephalometric teleradiography lateral norms in three different moments: (TO) presurgical, (T1) instantaneous postsurgical and (T2) postsurgical at least six months. Results and Conclusion: the results showed that the bimaxillary surgery causes meaningful facial changes and mainly, the counter-clockwise mandible rotation and consequently the occlusal plane angle reduction showed statistically stable, suggesting a reliable opinion in Class II standard facial surgeries with defective mandibular.
\end{abstract}

Key words: Cephalometry. Internal fixators. Osteotomy, Le Fort. Surgery, oral. Dental occlusion.

\section{REFERÊNCIAS}

1. ARPORNNMAELONG, P.; SHAND, J. M.; HEGGIE, A. A. Skeletal stability following maxillary impaction and mandibular advancement. Int J Oral Maxillofac Surg, Philadelphia, v. 33, no. 7, p. 656-663, Oct. 2004.

2. ATAOGLU, H. et al. Bimaxillary orthognathic surgery in a patient with long face: a case report. Int J Adult Orthodon Orthognath Surg, Chicago, v. 14, no. 4, p. 304-309, 1999.

3. BAILEY, L. J. et al. Stability following superior repositioning of the maxilla by Le Fort I osteotomy: five-year follow-up. Int J Adult Orthodon Orthognath Surg, Chicago, v. 9, no. 3, p. 163-173, 1994.

4. BELL, W. H.; PROFFIT, W. R.; WHITE, R. P. Surgical correction of dentofacial deformities. Philadelphia: W. B. Saunders, 1980. v. 1.

5. BELL, W. H. et al. Bone healing and revascularization after to- tal maxillary osteotomy. J Oral Surg, Chicago, v. 33, no. 4, p. 253-260, Apr.1975.

6. BISHARA, S. E.; CHU, G. W.; JACOBSEN, J. R. Stability of the Le Fort I one-pice maxillary osteotomy. Am J Orthod Dentofacial Orthop, St. Louis, v. 94, no. 3, p. 184-200, Sept. 1988.

7. BORSTLAP, W. A.; STOELINGA, P. J.; HOPPENREIJS, T. J.; VAN'T HOF, M. A. Stabilization of sagittal split advancement osteotomias with miniplates: a prospective, multicentre study with two-year follow-up. Part II. Radiographic parameters. Int J Oral Maxillofac Surg, Philadelphia, v. 33, no. 6, p. 535-542, Sept. 2004.

8. BRAMMER, J. et al. Stability after bimaxillary surgery to correct vertical maxillary excess and mandibular deficiency. J Oral Surg, Chicago, v. 38, no. 9, p. 664-670, Sept. 1980.

9. BURSTONE, C. J. et al. Cephalometrics for orthognathic surgery. J Oral Surg, Chicago, v. 36, no. 4, p. 269-277, Apr. 1978.

10. CASKEY, R. T.; TURPIN, D. L.; BLOOMQUIST, D. S. Stability of mandibular lengthening using bicortical screw fixation. Am J 
Orthod Dentofacial Orthop, St. Louis, v. 96 , no. 4, p. 320-326, Oct.1989.

11. CASTEIGT, J.; DEREUDRE, B. Surgical approach of a vertical problem: the long face syndrome. Rev Stomatol Chir Maxillofac, Paris, v. 85 , no. 3, p. 214-223, 1984

12. CHAMELLO, P. D.; WOLFORD, L. M.; BUSCHANG, P. H. Occlusal plane alteration in orthognathic surgery- part II: long-term stability of results. Am J Orthod Dentofacial Orthop, St. Louis, v. 106 , no. 4, p. 434-440, Oct. 1994

13. ELLIS III, E.; REYNOLDS, S.; CARLSON, D. S. Stability of the mandible following advancement: a comparison of three postsurgical fixation techniques. Am J Orthod Dentofacial Orthop, St. Louis, v. 42, no. 1, p. 38-49, July 1988.

14. EPKER, B. N.; FISH, L. C. Dentofacial deformities: integrated orthodontics and surgical correction. St. Louis: C. V. Mosby, 1986. p. 400-406.

15. EPKER, B. N.; STELLA, J. P.; FISH, L. C. Dentofacial deformities: integrated orthodontic and surgical correction. 2nd ed. St. Louis: Mosby Year Book, 1995. v.1, p. 430-463.

16. EPKER, B. N.; STELLA, J. P.; FISH, L. C. Dentofacial deformities: integrated orthodontic and surgical correction. 2nd ed. St. Louis: Mosby Year Book, 1996. v. 2, p. 844-848.

17. FALTIN JÚNIOR, K. Quando tratar um paciente Classe II ou III com Ortopedia Facial aliada à Ortodontia convencional e quando indicar a cirurgia ortognática. In: ARAUJO, A. Cirurgia ortognática. São Paulo: Ed. Santos, 1999. cap.7, p. 89-106.

18. FONSECA, A. M. Tratamento ortodôntico-cirúrgico. In: GREGORET, J. Ortodontia e cirurgia ortognática: diagnóstico e planejamento. São Paulo: Ed. Santos, 1999. Seção 6, cap. 20-24, p. 437-520.

19. HENNES, J. A. et al. Stability of simultaneous mobilization of the maxila and mandible utilizing internal rigid fixation. Int $\mathbf{J}$ Adult Orthodon Orthognath Surg, Lombard, v. 3, no. 4, p. 127-141, 1988.

20. ILG, J. P. Osteotomia total da maxila tipo Le Fort I. In: ARAÚJO, A. Cirurgia ortognática. São Paulo: Ed. Santos, 1999. cap. 8 , p.146-168.

21. IVE, J.; MCNEILL, W.; WEST, R. Mandibular advancement: skeletal and dental changes during fixation. J Oral Surg, Chicago, v. 35 , no. 11 , p. 881-886, Nov. 1977

22. JACOBSON, A. Radiographic cephalometry from basics to videoimaging. Carol Stream: Quintessence, 1995

23. LANGLADE, M. Cefalometria ortodôntica. São Paulo: Ed. Santos, 1993.

24. McNAMARA JÚNIOR, J. A. Um método de avaliação cefalométrica. Ortodontia, São Paulo, v. 23, n. 3, p. 79-92, set./dez. 1990.

25. McNEIL, R. W. Surgical-orthodontic correction of open bite malocclusion. Am J Orthod, St. Louis, v. 64, no. 1, p. 38-49, July 1973.

26. PROFFIT, W. R.; BELL, W. H. Open bite. In: BELL, W. H.; PROFFIT, W. R.; WHITE, R. P. (Ed.). Surgical correction of dentofacial deformities. Philadelphia: W. B. Saunders, 1980. p. $1111-1113$

27. PROFFIT, W. R.; PHILLIPS, C.; TURVEY, T. A. Stability following superior repositioning of the maxilla by Le Fort I osteotomy. Am J Orthod Dentofacial Orthop, St. Louis, v. 92, no. 2, p. 151-161, Aug. 1987.

28. PROFFIT, W. R.; TURVEY, T. A.; PHILLIPS, C. Orthognathic surgery: a hierarchy of stability. Int J Adult Orthodon Orthognath Surg, Chicago, v. 11, no. 3, p. 191-204, 1996.

29. PROFFIT, W. R. et al. Surgical versus orthodontic correction of skeletal Class II malocclusion in adolescents: effects and indications. Int J Adult Orthodon Orthognath Surg, Chicago, v. 7, no. 4, p. 209-220, 1992.

30. REYNOLDS, S. T.; ELLIS III, E.; CARLSON, D. S. Adaptation of the suprahyoid muscle complex to large mandibular advancements. J Oral Maxillofac Surg, Philadelphia, v. 46, no. 12, p. 1077-1085, Dec. 1988

31. SATROM, K. D.; SINCLAIR, P. M.; WOLFORD, L. M. The stability of double jaw surgery: a comparison of rigid versus wire fixation. Am J Orthod Dentofacial Orthop, St. Louis, v. 99, no. 6, p. 550-563, June 1991.

32. SCHENDEL, S. A.; CARLLOTI Jr., A. E. Variations of total vertical maxillary excess. J Oral Maxillofac Surg, Philadelphia, v. 43, no. 8, p. 590-596, Apr. 1985.

33. SCHENDEL, S. A.; EPKER, B. N. Results after mandibular advancement surgery: an analysis of 87 cases. J Oral Surg, Chicago, v. 38, no. 4, p. 265-282, Apr. 1980.

34. SCHENDEL, S. A. et al. The long face syndrome: vertical maxillary excess. Am J Orthod, St. Louis, v. 70, no. 4, p. 399-408, Nov. 1976.

35. SCHUDY, F. F. The rotation of the mandible resulting from growth: its implications in orthodontic treatment. Angle Orthod, Appleton, v. 35, no. 1, p. 36-50, Jan. 1965.

36. SMITH, V.; WILLIAMS, S.; STAPLEFORD, R. Rigid internal fixation and the effects on the temporomandibular joint and mastigatory system. A prospective study. Am J Orthod Dentofacial Orthop, St. Louis, v. 102, no. 6, p. 491-500, Dec. 1992.

37. STOKER, N. G.; EPKER, B. N. The posterior maxillary osteotomy: a retrospective study of treatment results. Int J Oral Surg, Copenhagem, v. 3, no. 3, p. 153-157, June 1974.

38. TURVEY, T. A. et al. Simultaneous superior repositioning of the maxilla and mandibular advancement. Am J Orthod Dentofacial Orthop, St. Louis, v. 94, no. 5, p. 372-381, Nov. 1988.

39. VAN SICKELS, J. E.; LARSEN, A. J.; THRASH, W. J. A retrospective study of relapse in rigidly fixated sagittal split osteotomies: contributing factors. Am J Orthod Dentofacial Orthop, St. Louis, v. 93, no. 5, p. 413-418, May 1988.

40. WELCH, T. B. Stability in the correction of dentofacial deformities: a comprehensive review. J Oral Maxillofac Surg, Philadelphia, v. 47, no. 11, p. 1142-1149, Nov. 1989.

41. WILL, L. A. et al. Condilar position following mandibular advancement: its relationship to relapse. J Oral Maxillofac Surg, Philadelphia, v. 40, no. 9, p. 578-588, Sept. 1984.

42. WOLFORD, L. M.; BENNETT, M. A.; RAFFERTY, C. G. Modification of the mandibular ramus sagittal split osteotomy. Oral Surg Oral Med Oral Pathol, St. Louis, v. 64, no. 2, p.146-155, Aug. 1987.

43. WOLFORD, L. M.: CHAMELLO, P. D.: HILLIARD, F. Occlusal plane alteration in orthognathic surgery. J Oral Maxillofac Surg, Philadelphia, v. 51, no. 7, p. 730-740, July 1993.

44. WOLFORD, L. M.; CHAMELLO, P. D.; HILLIARD, F. Occlusal plane alteration in orthognathic surgery- part I: Effects on function and esthetics. Am J Orthod Dentofacial Orthop, St. Louis, v. 106, no. 3, p. 304-316, Sept. 1994

45. YELLICH, G.M. et al. Muscular and mandibular adaptation after lengthening, detachment, and reattachment of the masseter muscle. J Oral Surg, Chicago, v. 39, no. 9, p. 656-665, Sept. 1981.

Endereço de correspondência

Karina E. Dela Coleta Pizzol

Rua Dr. Waldo Barbieri, 41, bloco 5 apto.83 - Jardim Paulistano

CEP: 14.810-273 - Araraquara / SP

E-mail:nkpizzol@ig.com.br 